\title{
Herr (Frau) Doktor, ich möchte sterben ...
}

\section{Samia Hurst}

Prof. Dr. med., Institut Ethique, Histoire, Humanités (iEH2), Faculté de Médecine, Genf

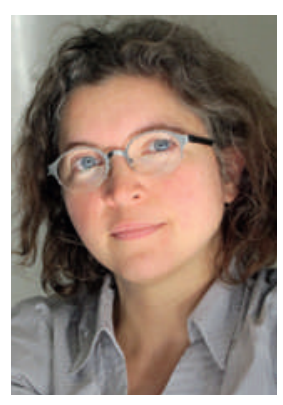

Wurden Sie schon einmal von einem Patienten gebeten, ihm beim Sterben zu helfen? Wenn ja, dann wissen Sie, dass dies in vielerlei Hinsicht ein Schlüsselmoment ist. Was ist in einem solchen Fall zu tun? Dies ist zunächst eine ganz persönliche, moralische Frage. Wenn keine selbstsüchtigen Beweggründe vorliegen und der Patient urteilsfähig ist, überlässt das Gesetz die Entscheidung für oder gegen die Sterbehilfe dem persönlichen Gewissen. Wie sollte man sich also in diesem Fall verhalten? In vielen Fällen weiss man es einfach nicht. Und wie verhalte ich mich als Arzt? Auch hier gibt es keine klare Antwort. Vor diesem Hintergrund stellt uns eine solche Bitte eines Menschen vor eine erhebliche Herausforderung.

Denn es handelt sich in der Tat um eine sehr schwierige und persönliche Frage. Allerdings dürfen wir dabei nicht vergessen, dass wir diesen Patienten gegenüber ganz klare Pflichten haben.

\section{Bleibt nach diesen Schritten das Verlangen nach Sterbehilfe bestehen, beginnt eine zweite Phase.}

Obwohl die Frage der Sterbehilfe durch die diesbezüglich herrschenden Kontroversen und Emotionen erschwert wird, müssen wir die Bitte des Patienten zunächst sehr bewusst und ohne Urteil zur Kenntnis nehmen. Wir helfen ihm nicht, indem wir das Vertrauen missbrauchen, das er uns durch das Aussprechen seines Wunsches entgegenbringt.

Bernacki RE, Block American College of Physicians High Value Care Task F. Communication about serious illness care goals: a review and synthesis of best practices. JAMA internal medicine. 2014;174(12):1994-2003 PubMed PMID: 25330167.

2 Schweizerische Akademie der Medizinischen Wissenschaften. Medizinisch-ethische Richtlinien: Betreuung von Patientinnen und Patienten am Lebensende. 2004

samia.hurst[at]unige.ch tivmedizin geltenden Modelle beurteilt werden. Es muss abgeklärt werden, ob eine Depression vorliegt, und diese muss dann behandelt werden. Dem Patienten muss eine palliativmedizinische Betreuung angeboten werden, sofern eine solche verfügbar ist. Diese auf Symptomkontrolle spezialisierte Disziplin beschränkt sich nicht auf die letzten Lebenstage eines Patienten, sie kann vielmehr bei jedem Patienten zum Tragen kommen, der ein schwer zu behandelndes Leiden hat.

Wenn im Anschluss daran die Bitte um Sterbehilfe bestehen bleibt und der Patient urteilsfähig ist, muss ein strukturiertes Gespräch über die weitere Vorgehensweise erfolgen [1]. Dem Patienten muss die Möglichkeit
Das Leiden des Patienten muss gemäss der in der Pallia- angeboten werden, eine Patientenverfügung zu verfassen. Wenn der Patient bereits lebenserhaltende Massnahmen erhält und er deren Fortsetzung ablehnt, so müssen diese eingestellt werden. Ist der Patient nicht urteilsfähig, müssen die Angehörigen in die Diskussion mit einbezogen werden. Die Planung der weiteren Behandlung spielt hier nach wie vor eine entscheidende Rolle, obwohl in diesem Fall natürlich die Sterbehilfe keine Option mehr darstellt.

Bleibt nach diesen Schritten das Verlangen nach Sterbehilfe bestehen, beginnt eine zweite Phase. An dieser Stelle kann jeder entscheiden, ob er das Verfahren fortsetzen oder abbrechen möchte. Die Schweizerische Akademie der Medizinischen Wissenschaften hat einen Leitfaden für die medizinische Praxis herausgegeben [2], zu dessen Anwendung jedoch niemand verpflichtet ist. In diesem Zusammenhang ist es aber wichtig zu berücksichtigen, dass es sich um eine zweite Phase handelt. Denn wir dürfen uns nicht durch unsere Unsicherheit in Bezug auf diese zweite Phase von den Schritten der ersten Phase abbringen lassen.

Der in zwei Phasen gegliederte Prozess verlangt von einigen unter uns auch ein Überdenken unserer Einstellung gegenüber den sehr schweizerischen Sterbehilfe-Organisationen. Diesen wird mitunter eine zu grosse Eile und mangelnde Sorgfalt im Hinblick auf die Explorationen vorgeworfen, die einer Sterbehilfe vorausgehen müssen. Aufgrund des Mangels an verlässlichen Fakten und der Vielzahl an Beteiligten ist es schwer zu sagen, inwiefern diese Kritik berechtigt ist. Sicher ist jedoch, dass die von den Vereinen vermeintlich vernachlässigten Schritte genau diejenigen sind, bei denen wir mitwirken sollten. Das Verlangen des Patienten ernst nehmen; nach anderen Möglichkeiten suchen, ihm zu helfen; seine Urteilsfähigkeit feststellen und ermitteln, welche Wünsche und Ängste er in Bezug auf sein Lebensende hat - all diese Aufgaben liegen in unserer Verantwortung. Und dabei spielt es keine Rolle, ob wir «Befürworter» oder «Gegner» der Sterbehilfe sind. Ein auf diesen Prinzipien basiertes Handeln, das selbst eine Interaktion mit den Sterbehilfe-Vereinen nicht ausschliesst, macht aus uns noch keine Mitwirkenden in der Sterbehilfe. 